\title{
Human cardiac extracellular matrix-chitosan-gelatin composite scaffold and its endothelialization
}

\author{
JINGJING LV ${ }^{1 *}$, WEI LIU ${ }^{2 *}$, GUOCHENG SHI ${ }^{1}$, FANG ZHU ${ }^{1}$, XIAOMIN HE ${ }^{1}$, \\ ZHONGQUN ZHU ${ }^{1}$ and HUIWEN CHEN ${ }^{1}$ \\ ${ }^{1}$ Heart Center, Shanghai Children's Medical Center, Shanghai Jiaotong University, School of Medicine, \\ Shanghai 200127; ${ }^{2}$ Department of Pediatric Cardiothoracic Surgery, Xinhua Hospital Affiliated \\ to Shanghai Jiaotong University, School of Medicine, Shanghai 200092, P.R. China
}

Received April 17, 2019; Accepted November 8, 2019

DOI: $10.3892 /$ etm.2019.8349

\begin{abstract}
The present study developed a cardiac extracellular matrix-chitosan-gelatin (cECM-CG) composite scaffold that can be used as a tissue-engineered heart patch and investigated its endothelialization potential by incorporating CD34 ${ }^{+}$ endothelial progenitor cells (EPCs). The cECM-CG composite scaffold was prepared by blending cardiac extracellular matrix (cECM) with biodegradable chitosan-gelatin (CG). The mixture was lyophilized using vacuum freeze-drying. CD34 ${ }^{+}$ EPCs were isolated and seeded on the scaffolds, and then the endothelialization effect was subsequently investigated. Effects of the scaffolds on CD34+ EPCs survival and proliferation were evaluated by immunofluorescence staining and MTT assay. Cell differentiation into endothelial cells and the influence of the scaffolds on cell differentiation were investigated by reverse transcription-quantitative PCR (RT-qPCR), immunofluorescence staining and tube formation assay. The present results indicated that most cells were removed after decellularization, but the main extracellular matrix components were retained. Scanning electron microscopy imaging illustrated three-dimensional and porous scaffolds. The present results suggested the cECM-CG composite scaffold had a higher water absorption ability compared with the CG scaffold. Additionally, compared with the CG scaffold, the cECM-CG composite scaffold significantly increased cell survival and proliferation, which suggested its non-toxicity and biocompatibility. Furthermore, RT-qPCR,
\end{abstract}

Correspondence to: Professor Huiwen Chen or Professor Zhongqun Zhu, Heart Center, Shanghai Children's Medical Center, Shanghai Jiaotong University, School of Medicine, 1678 Dongfang Road, Shanghai 200127, P.R. China

E-mail: chenhuiwen@scmc.com.cn

E-mail: zhuzhongqun@scmc.com.cn

*Contributed equally

Key words: cardiac extracellular matrix, scaffold, endothelial progenitor cells, heart patch, endothelialization immunofluorescence and tube formation assay results indicated that CD34+ EPCs differentiated into endothelial cells, and the cECM-CG composite scaffold promoted this differentiation process. In conclusion, the present results indicated that the human cECM-CG composite scaffold generated in the present study was a highly porous, biodegradable three-dimensional scaffold which supported endothelialization of seeded CD34+ EPCs. The present results suggested that this cECM-CG composite scaffold may be a promising heart patch for use in heart tissue engineering for congenital heart disease.

\section{Introduction}

Congenital heart disease (CHD) is one of the most common birth defects and is a major cause of childhood morbidity and mortality (1). Most patients with CHD require surgery to correct these heart defects (2). Some complex CHDs require multiple open-heart surgeries; consequently, numerous heart patches for the repair of cardiac defects are needed (2). Despite significant progress in the development of various materials to make cardiac patches, including Dacron (3), Gore-Tex (4) and autologous (5) or bovine pericardium (6), significant drawbacks exist, including increased risk of immune response (7), calcification $(6)$, thrombosis $(8,9)$ and the lack of growth potential (10). Moreover, these materials may become fibrous calcified tissues that cannot degrade, thereby preventing simultaneous growth within the heart and loss of function over time (11). Hence, patients typically require reoperation, which may cause delayed recovery and could result in an increased economic burden to both the family and society (11). Therefore, optimal materials for heart patches should be able to inhibit the immune response, calcification and thrombosis, and support growth of native tissue (10).

With the advantages of non-toxicity, biocompatibility and degradability, natural materials such as chitosan, collagen and gelatin have been used in various tissue engineering applications (12-14). These materials have shown good scaffold properties that allow for better mechanics and a significant influence on cellular behavior (12-14). However, the compositions of these materials are relatively simple and cannot provide a complete extracellular matrix (ECM) environment (15). On 
the other hand, ECM harvested from decellularized tissue lacks most of the immunogenicity but retains the majority of the ECM components (16). Previous studies showed that ECM, which is biocompatible, provides physical structural support and plays an essential role in providing signaling for cell attachment, proliferation and differentiation (17-19). Thus, the ideal biological scaffold would be made by a mixture of these two types of materials, and would be able to retain mechanical properties while also providing an extracellular matrix environment (20).

Previous studies found that ECM can be harvested from tissues such as cartilage, skeletal muscle, tendons, adipose tissue, vessels, lung, liver, intestine $(17,21,22)$ and bovine ureter (23), and may be applied as biological scaffolds with beneficial results. Previous studies in which porcine heart matrix was used for construction of tissue engineered heart patches demonstrated that porcine ECM could support cardiomyocytes, improved heart function and was capable of heart defect repair $(24,25)$. However, ECM is frequently derived from non-cardiac or heart tissue of other species. The present study hypothesized that ECM from human heart tissue may have a higher biocompatibility. Therefore, the present study combined human cardiac (cECM) with biodegradable natural chitosan-gelatin (CG) for the construction of cardiac extracellular matrix-chitosan-gelatin (cECM-CG) composite scaffold and studied its characteristics.

Thrombosis is a significant limitation of current grafts (8). Accumulating evidence showed that the endothelium, which maintains vessel integrity, plays an essential role in preventing thrombosis (26). Previous studies suggested that endothelial progenitor cells (EPCs), characterized by the ability to differentiate into endothelial cells, are a potential source of endothelial repair by participating in the process of endothelialization (27-29). Moreover, $\mathrm{CD} 34^{+}$cells are considered to be EPCs (30). A previous study showed that stent surfaces coated with antibodies against CD34, exhibited high affinity for EPC and could promote rapid endothelialization, prevent stent thrombosis and reduce restenosis (31). The present study hypothesized that $\mathrm{CD} 34^{+}$EPCs seeded on the scaffold of tissue-engineered heart patches could promote the scaffold endothelialization.

The present study created a cECM-CG composite scaffold seeded with $\mathrm{CD} 34^{+}$EPCs intended for tissue-engineered heart patch. The present study investigated the characteristics of this scaffold, including its endothelialization.

\section{Materials and methods}

Cardiac tissue and bone marrow samples. Human right atrial appendage and bone marrow samples were collected after written informed consent from parents of pediatric patients was obtained. All experiments were approved by the Ethics Committee at Shanghai Children's Medical Center (approval no. SCMCIRB-K2016025). All investigations were conducted according to the principles expressed in the Declaration of Helsinki. Specimens of right atrial appendage and bone marrow of sternum were collected from the patients (including 22 females and 28 males; age, 1 month- 3 years old) diagnosed as ventricular septal defect (VSD) or atrial septal defect (ASD) during open-heart surgery from May 2016 to May 2018 in our hospital. Right atrial appendages were processed for $\mathrm{CECM}$ preparation. Bone marrow was used to isolate $\mathrm{CD} 34^{+}$EPCs.

Preparation of cECM and scaffolds. Collected right atrial appendages were frozen in optimal cutting temperature compound, sectioned at $50 \mu \mathrm{m}$, and decellularized in $0.2 \%$ SDS solution for $12 \mathrm{~h}$ with constant shaking at $37^{\circ} \mathrm{C}$. Subsequently, the tissues were stirred in $200 \mathrm{mg} / 1$ DNase I solution for $30 \mathrm{~min}$ at $37^{\circ} \mathrm{C}$. Finally, the decellularized cardiac tissue was rinsed with deionized water until complete removal of detergents. The ECM was lyophilized, quantified and milled into fine powder (32). Then, $2 \%$ chitosan and $2 \%$ gelatin were prepared. Chitosan (cat. no. 1105508; Sigma-Aldrich; Merck) was added to $1 \%$ glacial acetic acid. The solution was shaken at $37^{\circ} \mathrm{C}$ for $12 \mathrm{~h}$ to ensure complete solubilization. Gelatin (cat. no. 1288485, Sigma-Aldrich; Merck) was dissolved in deionized water and incubated in a water bath at $50^{\circ} \mathrm{C}$ for $15 \mathrm{~min}$. Subsequently, chitosan and gelatin were mixed at a ratio of 2:1. The cECM powder was added to the chitosan and gelatin mixture solution, and was subsequently grounded using an ultrasonicator. The concentration of the cECM was $1.6 \mathrm{mg} / \mathrm{ml}$.

The CG mixture and the cECM-CG mixture were poured into a 96 -well plate $(50 \mu \mathrm{l} /$ well). Subsequently, both mixtures were frozen at $-80^{\circ} \mathrm{C}$ overnight, lyophilized for $24 \mathrm{~h}$ in a vacuum freeze-drier (VirtisBenchtop 6.6, SP Industries) to form porous scaffolds. The scaffolds were washed with ethanol to remove residual glacial acetic acid. Subsequently, the scaffolds were washed gently with PBS for five times (10 min each), and were sterilized by overnight incubation at room temperature using ultraviolet light in a cell culture hood.

Analysis of cECM components. Cardiac tissues before and after decellularization were fixed in $4 \%$ paraformaldehyde at $4^{\circ} \mathrm{C}$ for $24 \mathrm{~h}$, embedded in paraffin, and cut into $5-\mu \mathrm{m}$ thick sections. Sections were rinsed twice with xylene for $15 \mathrm{~min}$ and dehydrated with a descending alcohol series (100\% alcohol for $5 \mathrm{~min}$; 95\% alcohol for $5 \mathrm{~min}$; 75\% alcohol for $5 \mathrm{~min}$ ). Tissue was then stained with hematoxylin for $10 \mathrm{~min}$ and eosin for $5 \mathrm{~min}$ at room temperature.

The sections were dewaxed, rehydrated, stained in Weigert's iron hematoxylin solution for $5 \mathrm{~min}$ at room temperature, washed. Sections were then treated with Van Gieson's solution for 3-5 $\mathrm{min}$ at room temperature. Finally, the tissue was dehydrated, cleared and sealed.

The sections were dewaxed, rehydrated, stained in Weigert's iron hematoxylin solution at room temperature for $10 \mathrm{~min}$, rinsed and washed. Sections were then stained in Biebrich scarlet-acid fuchsin solution at room temperature for 10-15 min, washed and then differentiated in phosphomolybdic-phosphotungstic acid solution for 10-15 min. The sections were transferred directly to aniline blue solution and stained for 5-10 min at room temperature, rinsed and differentiated in $1 \%$ acetic acid solution for $2-5 \mathrm{~min}$ at room temperature. Finally, the sections were dehydrated, cleared and sealed. The presence of the adhesive proteins fibronectin and laminin in cECM was assessed by immunohistochemical staining. Briefly, the slides were placed in citrate antigen retrieval buffer and heated to $95^{\circ} \mathrm{C}$ for $20 \mathrm{~min}$. After cooling to 
room temperature, the sections were treated with $3 \%$ hydrogen peroxide solution for $20 \mathrm{~min}$ at room temperature and then incubated in a humidity chamber for $30 \mathrm{~min}$ with $10 \%(\mathrm{w} / \mathrm{v})$ normal goat serum (cat. no. 016201; Invitrogen; Thermo Fisher Scientific, Inc.). Hereafter, sections were incubated with rabbit anti-fibronectin antibody (1:250; cat. no. ab32419; Abcam) at $4^{\circ} \mathrm{C}$ overnight. After sections were washed with PBS, goat anti-rabbit IgG H\&L (HRP) (1:1,000; cat. no. ab6721; Abcam) was added for $30 \mathrm{~min}$. Sections were then rinsed in PBS, and diaminobenzidine solution (DAB) was applied. After staining, slides were dehydrated, cover-slipped. All sections were imaged under a light Olympus CX31 microscope (magnification x20; Olympus Corporation).

cECM quantification. Glycosaminoglycans (GAG) present in the cECM was measured using Glycosaminoglycans Assay kit (Chondrex, Inc). Briefly, samples before and after decellularization were weighed after lyophilization. Subsequently, lyophilized samples were digested and stained with 1 , 9-dimethylmethylene blue (DMB) for $4 \mathrm{~min}$ at room temperature. A dilution series of chondroitin sulfate was used as standard. Absorbance was measured at $525 \mathrm{~nm}$ with a Synergy HT Microplate Absorbance Reader (BioTek Instruments, Inc.). The GAG content was normalized to the dry weight of the samples.

DNA content was assayed using the tissue gDNA Miniprep kit (Biomiga, Inc.), and DNA concentration was determined by spectrophotometry (NanoDrop 2000c; Thermo Fisher Scientific, Inc.).

Determination of scaffold characteristics. Scanning electron microscope (SEM) was used to visualize the structure of the $\mathrm{CG}$ and cECM-CG scaffolds. Scaffolds were prepared by fixation with $2.5 \%$ glutaraldehyde for $2 \mathrm{~h}$ at room temperature, followed by dehydration with a series of ethanol rinses (30-100\%). The samples were critical point dried and coated with iridium. Electron microscopy images were obtained using SEM (magnification, x800; Quanta 2000; FEI; Thermo Fisher Scientific, Inc.).

CG and cECM-CG scaffolds were created in a 96-well plate in triplicates. The dry weight (Wd) of both scaffolds was measured before they were transferred to a 24-well plate and allowed to swell in PBS. The swelled weight (Ws) was measured every $10 \mathrm{~min}$ for $1 \mathrm{~h}$. Swelling ratios were calculated as follows: Swelling ratio $=(\mathrm{Ws}-\mathrm{Wd}) / \mathrm{Wd}$.

Isolation and culture of $C D 34^{+}$EPCs. Human bone marrow samples were collected from pediatric patients undergoing VSD or ASD surgery. Bone marrow mononuclear cells were collected using gradient centrifugation. Bone marrow was resuspended in Hank's balanced salt solution (GE Healthcare Life Sciences) and $20 \mu / \mathrm{ml}$ heparin (Sigma-Aldrich; Merck) and then centrifuged for $30 \mathrm{~min}$ at $400 \mathrm{~g}$ at room temperature. CD $34^{+}$EPCs were isolated from the mononuclear cells using magnetic cell sorting. Then the cells were seeded on the pre-prepared scaffolds at $37^{\circ} \mathrm{C}$ in $\mathrm{CO}_{2}$ incubator.

Flow cytometry analysis. To study the purity of sorted cells, freshly isolated CD $34^{+}$EPCs were collected for flow cytometry analysis. Briefly, cell suspensions were centrifuged at $300 \mathrm{x} \mathrm{g}$ for $5 \mathrm{~min}$ at $4^{\circ} \mathrm{C}$, and cell pellets resuspended in PBS containing 1\% BSA. Mouse Anti-Human CD34 antibody (cat. no. 560710; BD Biosciences) was diluted to 1:100 in PBS. Samples were incubated for $30 \mathrm{~min}$ in the dark on ice, followed by two washing steps with PBS (centrifugation for $5 \mathrm{~min}$ at $300 \mathrm{x} \mathrm{g}$ after each washing step at $4^{\circ} \mathrm{C}$ ). Finally, samples were subjected to flow cytometry analysis using Accuri C6 flow cytometer (BD Biosciences). Fluorescence was analyzed with CFlow Plus software (BD Biosciences).

Cell viability analysis. To investigate the viability of CD34 EPCs cultured on the scaffolds, cell viability was analyzed 5 days after seeding using a viability assay. Cells were collected, washed with PBS, and incubated in $5 \mu \mathrm{mol} / 1$ calcein AM (labeling living cells; Thermo Fisher Scientific, Inc.) and $9 \mu \mathrm{mol} / \mathrm{l}$ propidium iodide (labeling dead cells; Thermo Fisher Scientific, Inc.) for $30 \mathrm{~min}$ at $37^{\circ} \mathrm{C}$. Images of the stained samples were obtained using an optical microscope (Nikon Corporation). The number of living and dead cells was calculated based on three different fields of view (magnification, $\mathrm{x} 20)$.

Cell proliferation analysis. To identify the proliferative activity of $\mathrm{CD}_{34}{ }^{+} \mathrm{EPCs}$, Cells cultured on CG and cECM-CG scaffolds were stained with primary antibody Mouse anti-Ki67 IgG (1:200; cat. no. 561165; BD Biosciences, CA, USA) by overnight incubation at $4^{\circ} \mathrm{C}$. Donkey anti-mouse $\mathrm{IgG}$ conjugated to Alexa 488 (1:500; cat. no. R37114; Invitrogen; Thermo Fisher Scientific, Inc.) was added as the secondary antibody and incubated for $1 \mathrm{~h}$ at room temperature. Cells were counterstained with DAPI for $15 \mathrm{~min}$ at room temperature. For the negative controls, cells were incubated in 5\% donkey serum at room temperature (cat. no. 017-000-121; Jackson ImmunoResearch Laboratories, Inc.) instead of the primary antibody at $4^{\circ} \mathrm{C}$ overnight. Fluorescent images were acquired using a Nikon fluorescence microscope (magnification, x20; Nikon Corporation).

MTT assay. Cells were incubated with CellTiter96 ${ }^{\circledR}$ AQueous One Solution Reagent (Promega Corporation) at $37^{\circ} \mathrm{C}$ for $4 \mathrm{~h}$ after being cultured on CG and cECM-CG scaffolds for 1, 3, 5, 7, 10 and 14 days. The absorbance at $490 \mathrm{~nm}$ was measured using the Biotek Synergy HT Multi-Mode Microplate Reader (BioTek Instruments, Inc.).

Cell differentiation analysis and reverse transcription-quantitative PCR (RT-qPCR). A comparative RT-qPCR analysis was performed for the endothelial cell-associated genes CD31, von Willebrand factor (vWF) and CD144. Primer sequences are presented in Table I. Cells seeded on the CG and cECM-CG scaffolds were collected at day 21 and RNA was isolated using Quick-RNA MicroPrep (Zymo Research Corp.). Extracted RNA concentration was measured by spectrophotometry (NanoDrop 2000; Thermo Fisher Scientific, Inc.), and 400 ng RNA was used for first-strand cDNA synthesis (First-Strand cDNA Synthesis Kit; Yeasen Technologies). Subsequently, RT-qPCR was performed using SYBR (Hieff qPCR SYBR Green Master Mix, High Rox Plus; Yeasen Technologies). The amplification reactions were performed with initial denaturation at $95^{\circ} \mathrm{C}$ for $5 \mathrm{~min}$, followed by 40 cycles of two-step 
Table I. Primers for reverse transcription-quantitative PCR.

\begin{tabular}{ll}
\hline Gene & \multicolumn{1}{c}{ Primer sequence $\left(5^{\prime} \rightarrow 3^{\prime}\right)$} \\
\hline GAPDH & F: CTCATTTCCTGGTATGACAACGA \\
& R: CTTCCTCTTGTGCTCTTGCT \\
CD31 & F: AACAGTGTTGACATGAAGAGCC \\
& R: TGTAAAACAGCACGTCATCCTT \\
vWF & F: CCGATGCAGCCTTTCGGA \\
& R: TCCCCAAGATACACGGAGAGG \\
CD144 & F: AAGCGTGAGTCGCAAGAATG \\
& R: TCTCCAGGTTTTCGCCAGTG
\end{tabular}

vWF, von Willebrand factor; F, forward; R, reverse.

PCR at $95^{\circ} \mathrm{C}$ for $10 \mathrm{sec}$ and $60^{\circ} \mathrm{C}$ for $30 \mathrm{sec}$. Primer specificity was determined based on the melt curve analysis. The housekeeping gene GAPDH was chosen for normalization. Fold change expression was calculated using $2^{-\Delta \Delta C q}(33)$. $n=3$ for each condition.

Immunofluorescence staining. To further identify the differentiation of the CD34+ EPCs into endothelial cells, at day 21 cultured cells were fixed in $4 \%$ paraformaldehyde for $15 \mathrm{~min}$ at room temperature, permeabilized in $0.5 \%$ Triton X-100, followed by blocking with 5\% donkey serum (cat. no. 017-000-121; Jackson ImmunoResearch Laboratories, Inc.) for $1 \mathrm{~h}$ at room temperature and overnight incubation at $4{ }^{\circ} \mathrm{C}$ with the following primary antibodies: Mouse anti-CD31 (1:200; cat. no. ab24590; Abcam), rabbit anti-vWF (1:200; cat. no. ab6994; Abcam). Then, secondary antibodies of donkey anti-mouse Alexa594 (1:500; cat. no. A32744; Invitrogen; Thermo Fisher Scientific, Inc.) and donkey anti-rabbit Alexa488 (1:500; cat. no. A-21206; Invitrogen; Thermo Fisher Scientific, Inc.) were added to the samples and incubated for $1 \mathrm{~h}$ at room temperature. Cell nucleus was counterstained using DAPI for $15 \mathrm{~min}$ at room temperature. Fluorescence was observed using a fluorescence microscope (magnification, x40; Nikon Corporation).

In vitro tube formation assay. Tube formation assay was performed as described previously (34). Human umbilical vein endothelial cells (HUVECs; ScienCell Research Laboratories, Inc.) were serum starved overnight at $37^{\circ} \mathrm{C}$ in 1640 medium (cat. no. 72400120; Thermo Fisher Scientific, Inc.) without FBS. Matrigel (BD BioSciences) was thawed at $4^{\circ} \mathrm{C}$ overnight and added into the pre-chilled 96 -well plate using cooled pipette tips. Each well was coated homogeneously with $50 \mu 1$ Matrigel and incubated at $37^{\circ} \mathrm{C}$ with $5 \% \mathrm{CO}_{2}$ for $1 \mathrm{~h}$. Starved HUVECs were seeded at a density of $2 \times 10^{4} /$ well and cultured with a different conditioned medium for $8 \mathrm{~h}$ at $37^{\circ} \mathrm{C}$ with $5 \%$ $\mathrm{CO}_{2}$. The conditioned medium was collected from the CD34 EPCs cultured on the CG or cECM-CG scaffolds for $24 \mathrm{~h}$ in 1640 medium (cat. no. 72400120; Gibco; Thermo Fisher Scientific, Inc.) without FBS. Images were obtained using a phase-contrast light microscope (magnification, x20; Olympus Corporation). Tube-forming capacity was quantified based on the total length of capillary-like structures and the total number of branch points. The experiment was repeated three times, with duplicate measurements of each condition in each experiment.

Statistical analysis. Statistical analyses were performed using SPSS 13.0 (SPSS Inc.) and GraphPad Prism 5.0 software (GraphPad Prism Software, Inc.). Each experiment was repeated $\geq 3$ times. Data are presented as mean \pm SEM. Student's t-test was used to analyze the data. $\mathrm{P}<0.05$ was considered to indicate a statistically significant difference.

\section{Results}

Preparation and characterization of $c E C M$ and $C G / c E C M-C G$ scaffolds. To detect whether the decellularization process was complete and the main ECM components were retained, histologic and immunohistochemical analyses were performed. Histologic analysis showed that most of the cells were removed and the main cECM components were well preserved (Fig. 1A). Histology and immunohistochemistry analyses were used to evaluate the constructs of the cECM before and after decellularization (Fig. 1B-E). The components of the structural protein collagen (Fig. 1B) and elastic fiber (Fig. 1C) and the adhesive protein fibronectin (Fig. 1D) and laminin (Fig. 1E) present in the cECM were retained. DNA content analysis identified the complete decellularization process and removal of cell immunogenicity (Fig. 1F). Although the level of GAG was significantly decreased after decellularization, $\sim 18 \%$ of the GAG could be preserved after decellularization $(2.57 \pm 0.12 \mu \mathrm{g} / \mathrm{mg}$ before decellularization vs. $0.41 \pm 0.03 \mu \mathrm{g} / \mathrm{mg}$ after decellularization; Fig. $1 \mathrm{G})$.

The gross appearance of the newly lyophilized scaffolds was white and uniform (Fig. 1H). Microstructures of the scaffolds were assessed using scanning electron microscopy; representative images are presented in (Fig. 1I and J). Both scaffolds presented uniform three-dimensional structures and similar pore sizes of $40-100 \mu \mathrm{m}$, which is sufficient for cell ingrowth and distribution on multiple layers (35).

Water absorption ability, defined by the swelling ratio, was evaluated by comparing the dry and hydrated mass of CG and cECM-CG composite scaffolds. Both scaffolds presented a similar water uptake trend as both started swelling rapidly in the first $10 \mathrm{~min}$, indicating good water absorption and retention capacity (Fig. 1K). However, the cECM-CG composite scaffold absorbed water 10 times its dry weight, whereas the CG scaffold absorbed only about 5 times its dry weight. The present results suggested that the addition of cECM may lead to increased water absorption capacity.

cECM-CG composite scaffolds promote survival and proliferation of $\mathrm{CD}_{3} 4^{+} \mathrm{EPCS}$. Average purity of $\mathrm{CD} 34^{+} \mathrm{EPCs}$ isolated by MACS was $98 \%$ (and <99.2\%) based on flow cytometry (Fig. 2A and B). Moreover, cell viability assay demonstrated the viability of $\mathrm{CD} 34^{+}$EPCs cultured on the scaffolds at day 5. Cells growing on both CG and cECM-CG composite scaffolds showed no dead cells, indicating that the scaffolds were non-toxic (Fig. 2C).

Cells growing on the cECM-CG composite scaffold showed a significant increase in Ki67 expression level compared with 

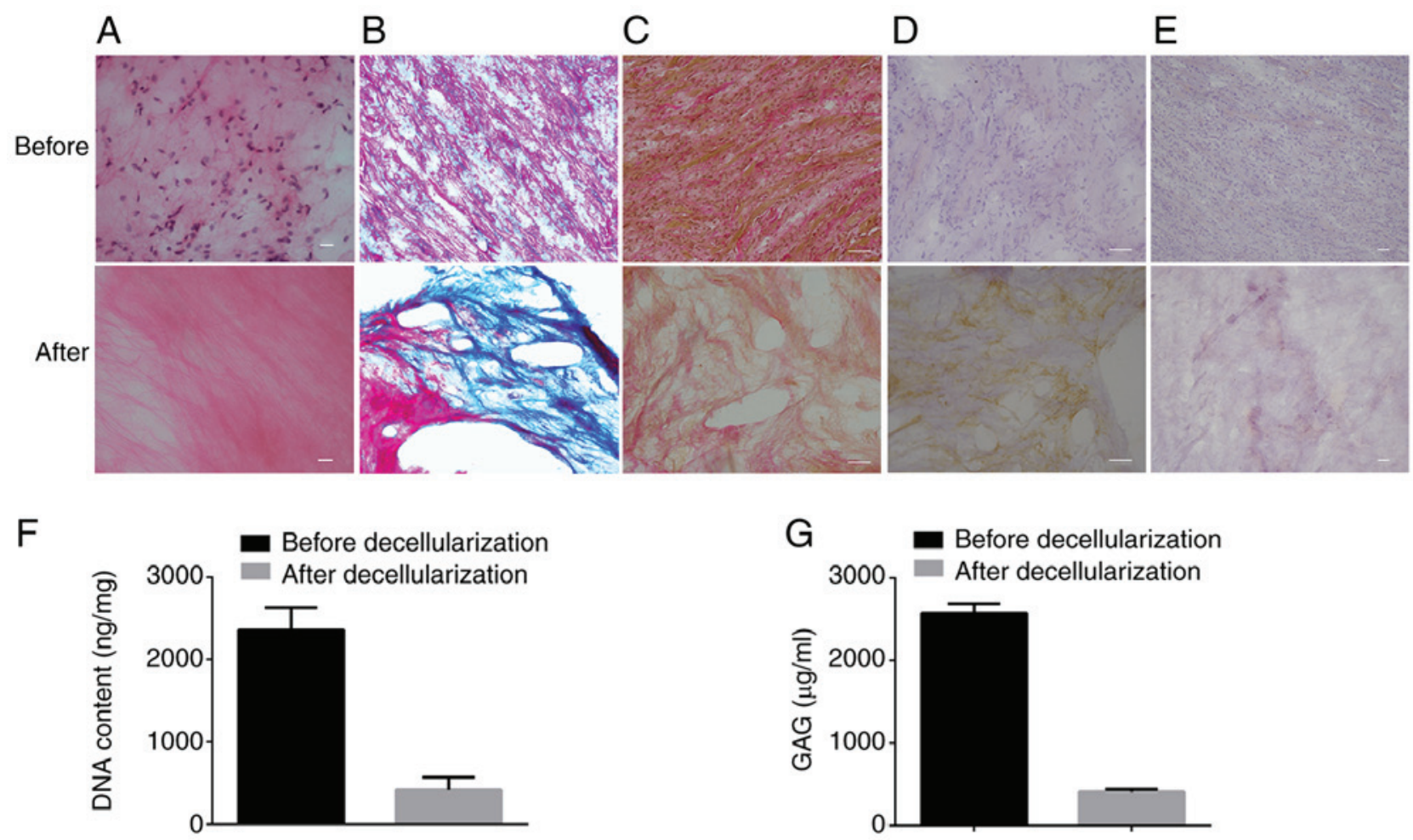

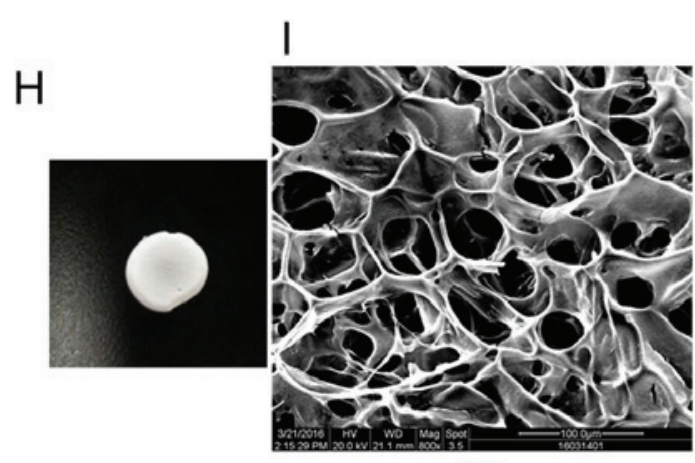

CG

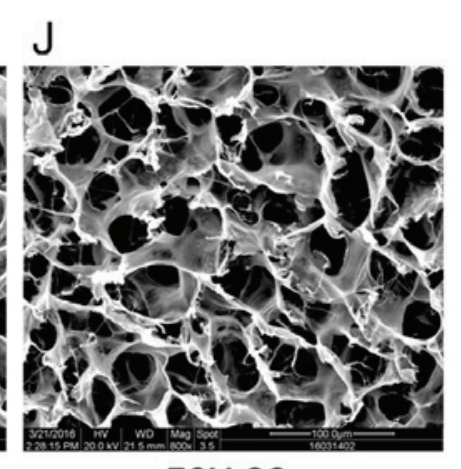

cECM-CG
$\mathrm{K}$

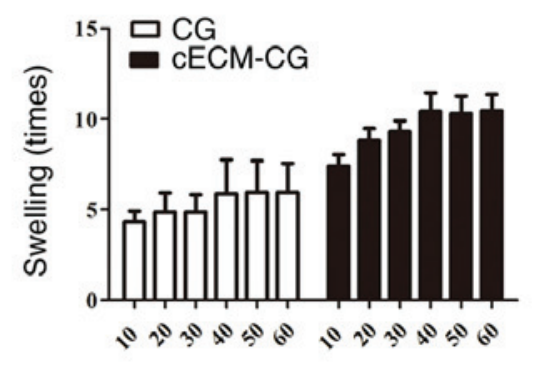

Time (min)

Figure 1. Preparation and characterization of cECM and CG/cECM-CG scaffolds. Comparison of cECM composition of heart tissue before and after decellularization. (A) Hematoxylin and eosin staining was performed to confirm complete decellularization. Scale bar, $10 \mu \mathrm{m}$. (B) Masson staining was performed for collagen fiber evaluation, collagen fibers were stained blue. Scale bar, $50 \mu \mathrm{m}$. (C) Van Gieson staining was performed for elastic fiber evaluation, elastic fibers were stained yellow. Scale bar, $50 \mu \mathrm{m}$. Immunohistochemical staining of (D) fibronectin and (E) laminin, fibronectin and laminin were stained brown. Scale bar, $50 \mu \mathrm{m}$. (F) Statistical analysis of DNA content. $\mathrm{n}=3$ in each group. (G) Statistical analysis of GAG content of heart tissue before and after decellularization. (H) Macrostructure of the scaffold. SEM analysis of (I) CG and (J) cECM-CG composite scaffolds. (K) Evaluation of the water absorption capacity of the scaffolds. GAG, glycosaminoglycans; cECM, cardiac extracellular matrix; CG, chitosan-gelatin; cECM-CG, cardiac extracellular matrix-chitosan-gelatin.

those growing on the CG scaffold (Fig. 2D and F), which suggested that the $\mathrm{CECM}-\mathrm{CG}$ composite scaffold could promote proliferation of $\mathrm{CD} 34^{+} \mathrm{EPCs}$ and had a better biocompatibility. Cell proliferation was also investigated by MTT assay on days 1, 3, 5, 7, 10 and 14. The proliferation of the CD34+ EPCs growing on the cECM-CG composite scaffold increased from day 1-14, reaching the maximal level on day 10. At all the time points, cECM-CG group exhibited a higher proliferation rate compared with the CG group (Fig. 2E). Therefore, the present results indicated that the $\mathrm{CECM}-\mathrm{CG}$ scaffold promoted proliferation of bone marrow-derived CD $34^{+}$EPCs.

cECM-CG composite scaffolds promote differentiation of $C D 34^{+} E P C s$. To investigate the differentiation capacity of CD $34^{+}$EPCs seeded on the scaffolds, cells were cultured on $\mathrm{CG}$ and cECM-CG composite scaffolds. After culturing for 21 days, RT-qPCR was performed. The present results indicated that the mRNA expression levels of CD31

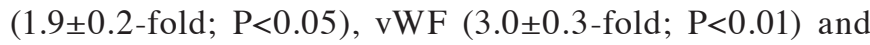
CD144 (9.8 \pm 0.1 -fold; $\mathrm{P}<0.001)$ of the $\mathrm{CD} 34^{+}$EPCs cultured on cECM-CG composite scaffold was significantly higher compared with cells cultured on CG scaffold (Fig. 3A). The differentiation of $\mathrm{CD} 34^{+}$EPCs cultured on the scaffolds was further investigated by immunofluorescence staining (Fig. 3D and E). At day 21, CD34 ${ }^{+}$EPCs cultured on cECM-CG scaffold showed a strong positive staining for endothelial marker CD31 and vWF compared with cells on CG scaffold. Differentiation rate of CD $34^{+}$EPCs cultured on the cECM-CG scaffold was significantly higher compared with the CG scaffold (Fig. 3B and C). The present results 

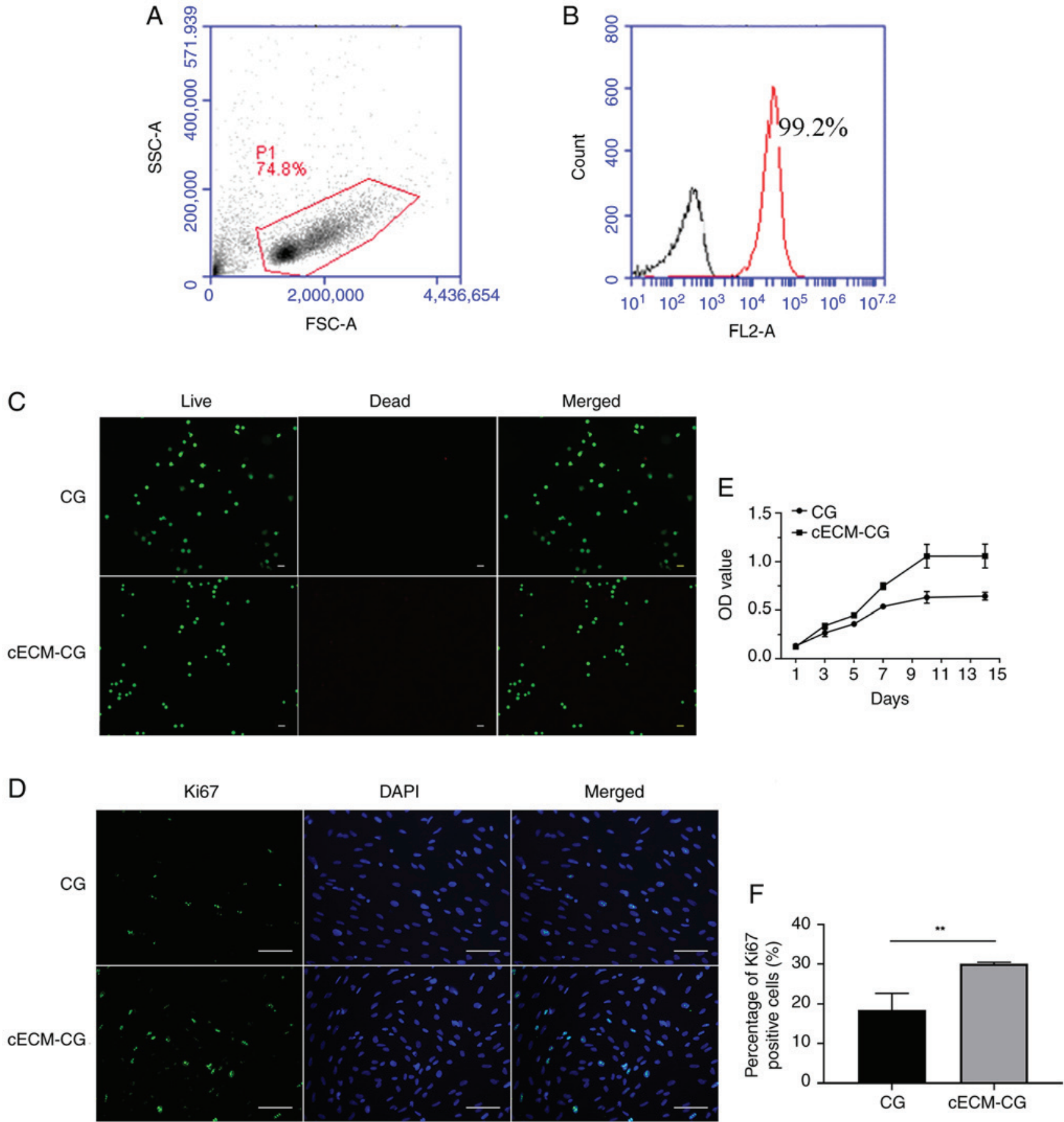

Figure 2. Scaffold composited with cECM improves cell survival and promotes proliferation of CD34+ EPCs. (A) The population of the sorted cells. (B) The purity of $\mathrm{CD} 34^{+}$EPCs after magnetic cell sorting isolation. (C) Cell viability assay analyzed the viability of CD $34^{+}$EPCs seeded on the scaffolds at day 5 by fluorescence microscopy. Living cells were stained green by calcein-AM. Dead cells were stained red by propidium iodide. Scale bar, $20 \mu \mathrm{m}$. (D) Representative images of cell proliferation assessed by Ki67 immunofluorescence staining at day 10. Positive cells were stained green. Scale bar, $100 \mu \mathrm{m}$. (E) Proliferation was analyzed by MTT assay on days 1, 3,5, 7, 10 and 14. $n=3$ in each group. (F) Percentage of Ki67 positive cells using immunofluorescence staining was calculated by counting the positive cells in three different and randomly chosen view fields. ${ }^{* * *} \mathrm{P}<0.01$ vs. CG. cECM, cardiac extracellular matrix; CG, chitosan-gelatin; cECM-CG, cardiac extracellular matrix-chitosan-gelatin; EPC, endothelial progenitor cells.

suggested that CD34+ EPCs can differentiate into endothelial cells and that the scaffold composited with cECM could promote this differentiation process.

cECM-CG composite scaffold-based conditioned medium increases tube formation of HUVECs. In addition to the direct differentiation of $\mathrm{CD} 34^{+}$EPCs into endothelial cells, the present study investigated whether the cECM-CG composite scaffold-based conditioned medium could enhance endothelialization. The present results indicated that $\mathrm{CECM}-\mathrm{CG}$ composite scaffold-based conditioned medium caused an increase in tube formation of HUVECs (Fig. 4A and B). Cells treated with a conditioned medium harvested from $\mathrm{CD} 34^{+}$ cells cultured on cECM-CG composite scaffold showed an increasing number of branch points (Fig. 4C). Furthermore, the tube length of the cECM-CG composite scaffold group 

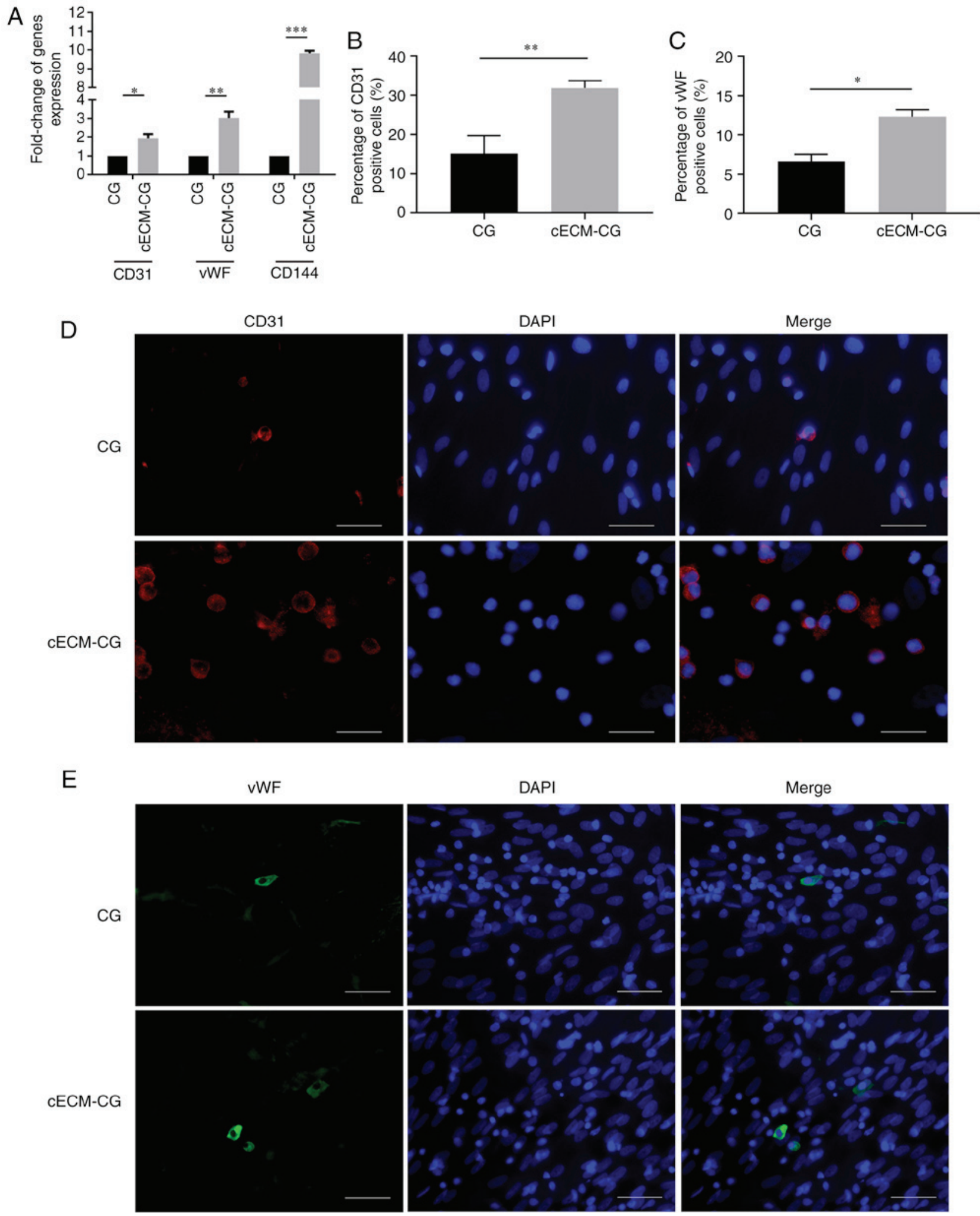

Figure 3. In vitro differentiation of CD34+ EPCs into endothelial cells cultured on CG and cECM-CG composite scaffolds. (A) Reverse transcription-quantitative PCR results showed that the CD34 EPCs seeded on cECM-CG scaffold upregulated the gene expression levels of EC markers including CD31, vWF and CD144 on day 21, compared with cells seeded on CG scaffold. Percentages of (B) CD31-positive and (C) vWF-positive cells were calculated at day 21 in three different and randomly chosen view fields. CD34 ${ }^{+}$EPCs on cECM-CG scaffold showed a higher differentiation rate compared with CG. The experiment was repeated three times. Representative images of immunofluorescence staining of the expression levels of (D) CD31 and (E) vWF. Scale bar, $50 \mu \mathrm{m}$. "P<0.05, ${ }^{* *} \mathrm{P}<0.01,{ }^{* * * *} \mathrm{P}<0.001$ vs. CG. cECM, cardiac extracellular matrix; CG, chitosan-gelatin; cECM-CG, cardiac extracellular matrix-chitosan-gelatin; EPC, endothelial progenitor cells; vWF, von Willebrand factor.

showed a significant increase compared with the CG scaffold group (Fig. 4D). The present results indicated that the
cECM-CG composite scaffold seeded with CD $34^{+}$EPCs could promote tube formation of the HUVECs. 

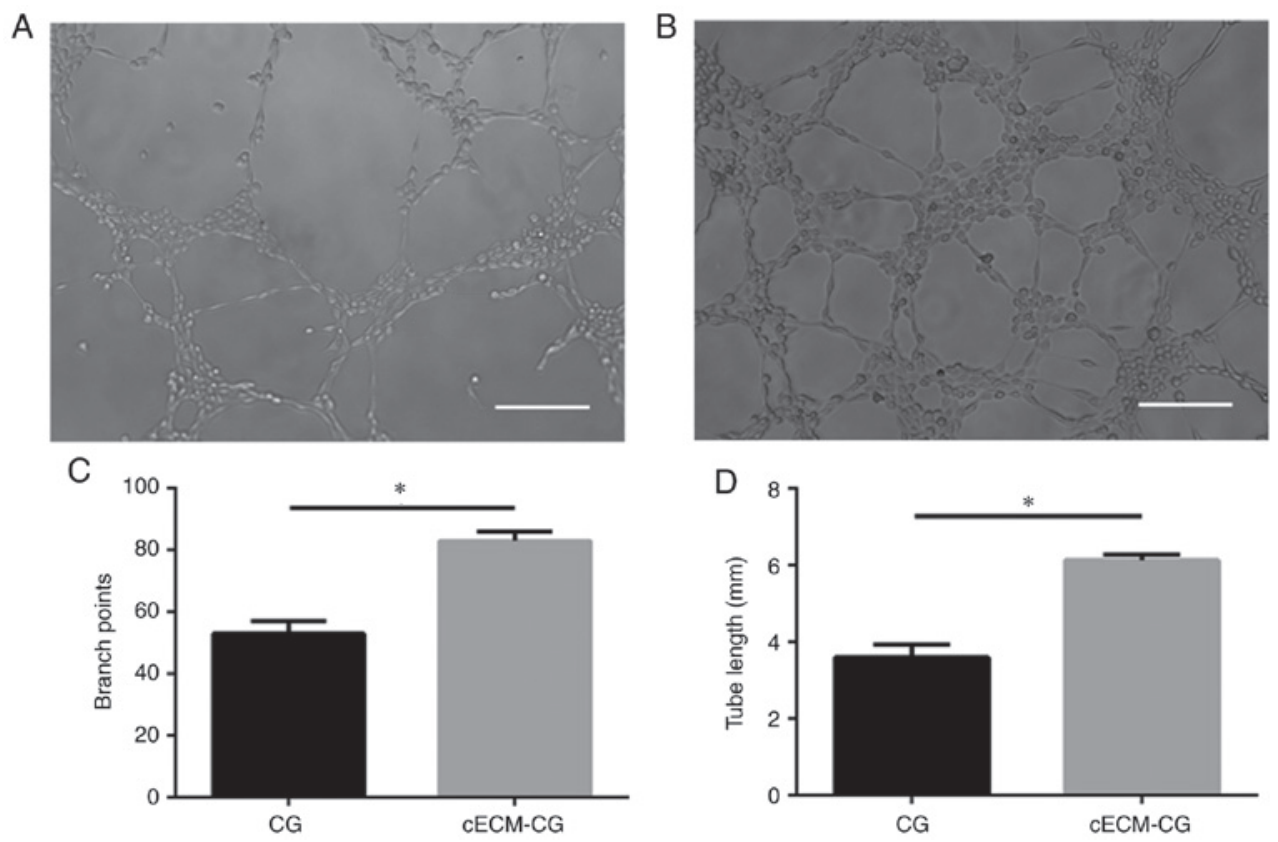

Figure 4. cECM-CG composite scaffold-based conditioned medium increases tube formation of HUVECs. (A and B) Representative images of the tube formation capacity of HUVECs induced by conditioned medium from CD34 cells cultured on (A) CG and (B) cECM-CG scaffolds. Scale bar, $100 \mu \mathrm{m}$. Quantitative analysis of the (C) branch points and (D) tube length of both groups. "P<0.05 vs. CG. CG, chitosan-gelatin; cECM-CG, cardiac extracellular matrix-chitosan-gelatin; EPC, endothelial progenitor cells; vWF, von Willebrand factor; HUVECs, human umbilical vein endothelial cells.

\section{Discussion}

The present study constructed a three-dimensional scaffold for tissue-engineered heart patch using human cECM, chitosan and gelatin. In addition, the present study investigated the characteristics and the endothelialization potential of the scaffold seeded with CD $34^{+}$EPCs.

ECM, previously described as containing various groups of molecules forming a microenvironment and providing structural and biological support for cells, has been reported to be associated with tissue remodeling and mechanical function (3). The composition of the ECM consists of a mixture of various molecules which form a three-dimensional matrix (16). In previous studies, some ECM components such as collagen and elastin, were used for the construction of cardiac grafts to repair heart defects $(36,37)$. Other studies used natural materials, such as chitosan and gelatin, for cardiac tissue engineering $(14,38)$. However, due to their relatively simple composition, natural materials cannot fully mimic the composition and complex structure of the ECM (15). Previous studies have attempted to use decellularized ECM from different tissues, such as porcine-derived cECM and intestinal submucosal or adipose-derived ECM, as a scaffold for tissue engineering $(18,24,39,40)$. Removal of cells expressing surface antigens leads to a significant reduction of immunogenicity (41). However, most of the ECM materials are derived from non-cardiac tissues or other species. Although ECM components are similar in many tissues, each tissue has its own distinct combination of molecules and has tissue-specific ECM architecture (42). Therefore, to the best of our knowledge, the most suitable tissue is human-derived heart tissue to develop a tissue-engineered heart patch for clinical use.

In the present study, specimens of discarded human right atrial appendage were collected during heart surgery of pediatric patients with CHD. These tissue samples can be obtained regularly in hospitals and conveniently cryopreserved. Following mixing the human cardiac-derived ECM with chitosan and gelatin, the mixture was lyophilized and was used to develop a cECM-CG composite scaffold for use as a tissue-engineered heart patch. The present results indicated that the scaffold was a three-dimensional porous scaffold that could promote cell survival and proliferation of $\mathrm{CD} 34^{+}$EPCs. More importantly, the present results suggested that the cECM-CG composite scaffold could promote the differentiation of $\mathrm{CD} 34^{+}$ EPCs, thus inducing the endothelialization of the scaffold. Decellularization of tissues involves a combination of physical, ionic, chemical and enzymatic processes (43). Importantly the decellularization agents should be tailored to different tissues. A previous study showed that the use of $1 \%$ SDS, an ionic agent, damaged the ECM components and negatively influenced their mechanical property (44). The present study decreased the concentration of SDS to $0.2 \%$. The present results indicated that the main components of the ECM, such as the structural proteins collagen and elastic fiber and the adhesive proteins fibronectin and laminin, were well maintained, while most of the cells and DNA were removed after decellularization. The present results suggested that the proteins in the $\mathrm{CECM}$, which mimicked the natural ECM structure and environment, were well preserved and the potential immunogenicity was mostly removed. Moreover, a scaffold should have a large surface area and be highly porous to ensure cell adhesion and transport of nutrients and metabolic wastes (45). The present cECM-CG composite scaffold was porous with a uniform pore size of $40-100 \mu \mathrm{m}$. The present scaffold had a strong water absorption capacity which may indicate high cell adhesion and proliferation, as well as exchange of substances between cells and the extracellular environment. Therefore, the present results suggested the cECM-CG composite scaffold could not only mimic the ECM environment but also indicated advantageous physical properties of porosity 
and efficient water absorption. Although human derived heart tissues may be the most suitable tissue for the engineering of a heart patch for clinical use, the heart tissues derived from humans were not sufficient to be used for the construction of the scaffold after decellularization. Therefore, the present study could not achieve scaffold for patient-specific use.

Thrombosis is a significant limitation of current grafts, and accumulating evidence shows that endothelial cells play an essential role in preventing thrombosis by maintaining endothelium integrity (46). CD $34^{+}$cells were shown to be endothelial progenitor cells with the capability to differentiate into endothelial cells, thus participating in endothelialization (30). Previous studies have shown that seeding of $\mathrm{CD} 34^{+}$cells on grafts enhances endothelialization of the grafts (47). A previous study using a EPC CD34+ capture stent coated with CD34 antibodies used for congenital heart disease surgery demonstrated that the stent could capture EPCs, thereby inhibiting thrombosis by promoting endothelial formation (48). In the present study, human bone marrow-derived $\mathrm{CD} 34^{+}$EPCs were selected and seeded on CG and cECM-CG scaffolds. The present results suggested that $\mathrm{CD} 34^{+}$EPCs cultured on cECM-CG composite scaffold showed increased mRNA expression levels of endothelial cell markers CD31, vWF and CD144 at day 21. CD144 is often used as a marker to identify endothelial cells (49). Although freshly isolated bone marrow-derived CD $34^{+}$endothelial progenitor cells can also express a small amount of CD144, $\mathrm{CD} 4^{+}$endothelial progenitor cells acquire higher expression of CD144 when differentiating into endothelial cells. As shown in our previous study, the expression ratio of CD144 in freshly isolated bone marrow-derived $\mathrm{CD}_{3} 4^{+}$endothelial progenitor cells was only $1 \%$ (49). In present immunofluorescence staining and tube formation assay suggested that the cECM-CG composite scaffold promoted the differentiation of $\mathrm{CD} 34^{+} \mathrm{EPCs}$ and enhanced tube formation. The present results indicated that the combination of $\mathrm{CD} 34^{+}$EPCs and cECM-CG composite scaffold could facilitate endothelialization of the scaffold. The achievement of endothelialization of grafts is thought to be critically important for the prevention of graft thrombosis (50). Future studies should include testing of the characteristics of the present scaffold in vivo, which could lead to further improvements of the material.

In conclusion, the present results suggested that the constructed cECM-CG composite scaffold provided advantageous properties and promoted cell survival and proliferation. Furthermore, seeding of CD $34^{+}$EPCs enhanced endothelialization of the scaffold. The present results indicated that the cECM-CG composite scaffold may potentially be used in heart tissue engineering research.

\section{Acknowledgements}

Not applicable.

\section{Funding}

The present study was supported by Shanghai Hygiene Science Research (grant no. 20134026), Shanghai Science and Technology Commission (grant nos. 14411964900 and 16411962700) and National Natural Science Foundation of China (grant no. 81670464).

\section{Availability of data and materials}

The datasets used and/or analyzed during the current study are available from the corresponding author on reasonable request.

\section{Authors' contributions}

$\mathrm{HC}$ and $\mathrm{ZZ}$ designed and supervised the experiments. JL and WL performed the experiments. JL, GS, FZ, and XH analyzed the data. JL wrote the article. All authors read and approved the final manuscript.

\section{Ethics approval and consent to participate}

All experiments were approved by the Ethics Committee at Shanghai Children's Medical Center (approval no. SCMC IRB-K2016025). Written informed consent from parents of pediatric patients was obtained.

\section{Patient consent for publication}

Not applicable.

\section{Competing interests}

The authors declare that they have no competing interests.

\section{References}

1. Benjamin EJ,MuntnerP,Alonso A,Bittencourt MS,Callaway CW, Carson AP, Chamberlain AM, Chang AR, Cheng S, Das SR, et al: Heart disease and stroke statistics-2019 update: A report from the American heart association. Circulation 139: e56-e528, 2019.

2. Tsilimigras DI, Oikonomou EK, Moris D, Schizas D, Economopoulos KP and Mylonas KS: Stem cell therapy for congenital heart disease: A systematic review. Circulation 136: 2373-2385, 2017.

3. Kochupura PV, Azeloglu EU, Kelly DJ, Doronin SV, Badylak SF, Krukenkamp IB, Cohen IS and Gaudette GR: Tissue-engineered myocardial patch derived from extracellular matrix provides regional mechanical function. Circulation 112 (Suppl 9): I144-I149, 2005.

4. Mosala Nezhad Z, Poncelet A, Fervaille C and Gianello P: Comparing the host reaction to CorMatrix and different cardiac patch materials implanted subcutaneously in Growing Pigs. Thoracic Cardiovasc Surg 67: 44-49, 2019.

5. Nishida H, Nakatsuka D, Kawano Y, Hiraiwa N, Takanashi S and Tabata M: Outcomes of totally endoscopic atrial septal defect closure using a glutaraldehyde-treated autologous pericardial patch. Circ J 81: 689-693, 2017

6. Lee C, Lim HG, Lee CH and Kim YJ: Effects of glutaraldehyde concentration and fixation time on material characteristics and calcification of bovine pericardium: Implications for the optimal method of fixation of autologous pericardium used for cardiovascular surgery. Interact Cardiovasc Thorac Surg 24: 402-406, 2017.

7. Rieder E, Steinacher-Nigisch A and Weigel G: Human immune-cell response towards diverse xenogeneic and allogeneic decellularized biomaterials. Int J Surg 36: 347-351, 2016.

8. Spadaccio C, Chello M, Trombetta M, Rainer A, Toyoda Y and Genovese JA: Drug releasing systems in cardiovascular tissue engineering. J Cell Mol Med 13: 422-439, 2009.

9. Anderson DEJ, Truong KP, Hagen MW, Yim EKF and Hinds MT: Biomimetic modification of poly(vinyl alcohol): Encouraging endothelialization and preventing thrombosis with antiplatelet monotherapy. Acta Biomater 86: 291-299, 2019.

10. Shoji T and Shinoka T: Tissue engineered vascular grafts for pediatric cardiac surgery. Transl Pediatr 7: 188-195, 2018.

11. Mantakaki A, Fakoya AOJ and Sharifpanah F: Recent advances and challenges on application of tissue engineering for treatment of congenital heart disease. PeerJ 6: e5805, 2018. 
12. Afewerki S, Sheikhi A, Kannan S, Ahadian S and Khademhosseini A: Gelatin-polysaccharide composite scaffolds for 3D cell culture and tissue engineering: Towards natural therapeutics. Bioeng Transl Med 4: 96-115, 2018.

13. Thein-Han WW, Saikhun J, Pholpramoo C, Misra RD and Kitiyanant Y: Chitosan-gelatin scaffolds for tissue engineering: Physico-chemical properties and biological response of buffalo embryonic stem cells and transfectant of GFP-buffalo embryonic stem cells. Acta Biomater 5: 3453-3466, 2009.

14. Pok S, Myers JD, Madihally SV and Jacot JG: A multilayered scaffold of a chitosan and gelatin hydrogel supported by a PCL core for cardiac tissue engineering. Acta Biomater 9: 5630-5642, 2013.

15. Silva AC, Rodrigues SC, Caldeira J, Nunes AM, Sampaio-Pinto V, Resende TP, Oliveira MJ, Barbosa MA, Thorsteinsdóttir S, Nascimento DS and Pinto-do-Ó P: Three-dimensional scaffolds of fetal decellularized hearts exhibit enhanced potential to support cardiac cells in comparison to the adult. Biomaterials 104: 52-64, 2016.

16. Yi S, Ding F, Gong L and Gu X: Extracellular matrix scaffolds for tissue engineering and regenerative medicine. Curr Stem Cell Res Ther 12: 233-246, 2017.

17. Scholl FG, Boucek MM, Chan KC, Valdes-Cruz L and Perryman R: Preliminary experience with cardiac reconstruction using decellularized porcine extracellular matrix scaffold: Human applications in congenital heart disease. World J Pediatr Congenit Heart Surg 1: 132-136, 2010.

18. Wang X, Yu T, Chen G, Zou J, Li J and Yan J: Preparation and characterization of a Chitosan/Gelatin/Extracellular Matrix Scaffold and its application in tissue engineering. Tissue Eng Part C Methods 23: 169-179, 2017.

19. Tian Y and Liu Y: Preparation and evaluation of extracellular matrix scaffold of human adipose tissue. Zhonghua Zheng Xing Wai Ke Za Zhi 33: 129-135, 2017 (In Chinese).

20. Stoppel WL, Hu D, Domian IJ, Kaplan DL and Black LD III: Anisotropic silk biomaterials containing cardiac extracellular matrix for cardiac tissue engineering. Biomed Mater 10: 034105, 2015.

21. Shell DH IV, Croce MA, Cagiannos C, Jernigan TW, Edwards N and Fabian TC: Comparison of small-intestinal submucosa and expanded polytetrafluoroethylene as a vascular conduit in the presence of gram-positive contamination. Ann Surg 241: 995-1004, 2005

22. Porzionato A, Stocco E, Barbon S, Grandi F, Macchi V and De Caro R: Tissue-engineered grafts from human decellularized extracellular matrices: A systematic review and future perspectives. Int J Mol Sci 19: pii: E4117, 2018.

23. Chemla ES and Morsy M: Randomized clinical trial comparing decellularized bovine ureter with expanded polytetrafluoroethylene for vascular access. Br J Surg 96: 34-39, 2009.

24. Pok S, Benavides OM, Hallal P and Jacot JG: Use of myocardial matrix in a chitosan-based full-thickness heart patch. Tissue Eng Part A 20: 1877-1887, 2014

25. Pok S, Stupin IV, Tsao C, Pautler RG, Gao Y, Nieto RM, Tao ZW, Fraser CD Jr, Annapragada AV and Jacot JG: Full-thickness heart repair with an engineered multilayered myocardial patch in rat model. Adv Healthc Mater: 6, 2017. doi: 10.1002/adhm.201600549.

26. Bunting S, Moncada S and Vane JR: Antithrombotic properties of vascular endothelium. Lancet 2: 1075-1076, 1977.

27. Walter DH, Rittig K, Bahlmann FH, Kirchmair R, Silver M, Murayama T, Nishimura H, Losordo DW, Asahara T and Isner JM: Statin therapy accelerates reendothelialization: A novel effect involving mobilization and incorporation of bone marrow-derived endothelial progenitor cells. Circulation 105: 3017-3024, 2002.

28. Urbich C and Dimmeler S: Endothelial progenitor cells: Characterization and role in vascular biology. Circ Res 95: 343-353, 2004

29. Werner N, Priller J, Laufs U, Endres M, Böhm M, Dirnagl U and Nickenig G: Bone marrow-derived progenitor cells modulate vascular reendothelialization and neointimal formation: Effect of 3-hydroxy-3-methylglutaryl coenzyme a reductase inhibition. Arterioscler Thromb Vasc Biol 22: 1567-1572, 2002.

30. Hirashima M, Kataoka H, Nishikawa S, Matsuyoshi $\mathrm{N}$ and Nishikawa S: Maturation of embryonic stem cells into endothelial cells in an in vitro model of vasculogenesis. Blood 93: 1253-1263, 1999.
31. Aoki J, Serruys PW, van Beusekom H, Ong AT, McFadden EP, Sianos G, van der Giessen WJ, Regar E, de Feyter PJ, Davis HR, et al: Endothelial progenitor cell capture by stents coated with antibody against CD34: The HEALING-FIM (healthy endothelial accelerated lining inhibits neointimal growth-first in man) registry. J Am Coll Cardiol 45: 1574-1579, 2005.

32. French KM, Boopathy AV, DeQuach JA, Chingozha L, Lu H, Christman KL and Davis ME: A naturally derived cardiac extracellular matrix enhances cardiac progenitor cell behavior in vitro. Acta Biomater 8: 4357-4364, 2012.

33. Livak KJ and Schmittgen TD: Analysis of relative gene expression data using real-time quantitative PCR and the 2(-Delta Delta C(T)) method. Methods 25: 402-408, 2001.

34. Olmer R, Engels L, Usman A, Menke S, Malik MNH, Pessler F, Göhring G, Bornhorst D, Bolten S, Abdelilah-Seyfried S, et al: Differentiation of human pluripotent stem cells into functional endothelial cells in scalable suspension culture. Stem Cell Reports 10: 1657-1672, 2018.

35. Zhang Y, Fan W, Ma Z, Wu C, Fang W, Liu G and Xiao Y: The effects of pore architecture in silk fibroin scaffolds on the growth and differentiation of mesenchymal stem cells expressing BMP7. Acta Biomater 6: 3021-3028, 2010.

36. Miyagi Y, Chiu LL, Cimini M, Weisel RD, Radisic M and Li RK: Biodegradable collagen patch with covalently immobilized VEGF for myocardial repair. Biomaterials 32: 1280-1290, 2011.

37. Shi C, Li Q, Zhao Y, Chen W, Chen B, Xiao Z, Lin H, Nie L, Wang D and Dai J: Stem-cell-capturing collagen scaffold promotes cardiac tissue regeneration. Biomaterials 32: 2508-2515, 2011

38. Fu JH, Zhao M, Lin YR, Tian XD, Wang YD, Wang ZX and Wang LX: Degradable chitosan-collagen composites seeded with cells as tissue engineered heart valves. Heart Lung Circ 26: 94-100, 2017.

39. Ropcke DM, Ilkjaer C, Tjornild MJ, Skov SN, Ringgaard S, Hjortdal VE and Nielsen SL: Small intestinal submucosa tricuspid valve tube graft shows growth potential, remodelling and physiological valve function in a porcine model $\uparrow$. Interact Cardiovasc Thorac Surg 24: 918-924, 2017.

40. Efraim Y, Sarig H, Cohen Anavy N, Sarig U, de Berardinis E, Chaw SY, Krishnamoorthi M, Kalifa J, Bogireddi $\mathrm{H}$, Duc TV, et al: Biohybrid cardiac ECM-based hydrogels improve long term cardiac function post myocardial infarction. Acta Biomater 50: 220-233, 2017.

41. Meyer SR, Nagendran J, Desai LS, Rayat GR, Churchill TA, Anderson CC, Rajotte RV,Lakey JR and Ross DB: Decellularization reduces the immune response to aortic valve allografts in the rat. J Thorac Cardiovasc Surg 130: 469-476, 2005.

42. Padhi A and Nain AS: ECM in differentiation: A review of matrix structure, composition and mechanical properties. Ann Biomed Eng: Sep 4, 2019. doi: 10.1007/s10439-019-02337-7 (Epub ahead of print)

43. Brown BN and Badylak SF: Extracellular matrix as an inductive scaffold for functional tissue reconstruction. Transl Res 163: 268-285, 2014.

44. Mancuso L, Gualerzi A, Boschetti F, Loy F and Cao G: Decellularized ovine arteries as small-diameter vascular grafts. Biomed Mater 9: 045011, 2014.

45. Hutmacher DW: Scaffolds in tissue engineering bone and cartilage. Biomaterials 21: 2529-2543, 2000.

46. Vane JR and Botting RM: Endothelium-thromboresistance. Wien Klin Wochenschr 103: 403-404, 1991.

47. Bhattacharya V, McSweeney PA, Shi Q, Bruno B, Ishida A, Nash R, Storb RF, Sauvage LR, Hammond WP and Wu MH: Enhanced endothelialization and microvessel formation in polyester grafts seeded with CD34(+) bone marrow cells. Blood 95: $581-585,2000$.

48. Cabanelas N, Martins JD and Pinto F: Initial use of endothelial progenitor cells capturing stents in paediatric congenital heart disease. Cardiol Young 24: 900-904, 2014.

49. Gao LP, Du MJ, Lv JJ, Schmull S, Huang RT and Li J: Use of human aortic extracellular matrix as a scaffold for construction of a patient-specific tissue engineered vascular patch. Biomed Mater 12: 065006, 2017.

50. Melchiorri AJ, Hibino N and Fisher JP: Strategies and techniques to enhance the in situ endothelialization of small-diameter biodegradable polymeric vascular grafts. Tissue Eng Part B Rev 19: 292-307, 2013

This work is licensed under a Creative Commons Attribution-NonCommercial-NoDerivatives 4.0 International (CC BY-NC-ND 4.0) License. 